\title{
New CUORICINO Results and Status of CUORE*
}

O. Cremonesi ${ }^{1) * *}$, R. Ardito ${ }^{1), 2)}$, C. Arnaboldi ${ }^{1)}$, D. R. Artusa ${ }^{3)}$, F. T. Avignone III'), M. Balata ${ }^{4)}$, I. Bandac ${ }^{3)}$, M. Barucci( ${ }^{5)}$, J. W. Beeman ${ }^{6)}$, F. Bellini ${ }^{7}$, C. Brofferio ${ }^{1)}$, C. Bucci ${ }^{4}$, S. Capelli ${ }^{1)}$, F. Capozzi ${ }^{1)}$, L. Carbone ${ }^{1)}$, S. Cebrian ${ }^{8)}$, M. Clemenza ${ }^{1}$, C. Cosmelli ${ }^{7)}$, R. J. Creswick ${ }^{3)}$, I. Dafinei ${ }^{7)}$, A. de Waard ${ }^{9)}$, M. Dolinski ${ }^{6),}$ 10), H. A. Farach ${ }^{3)}$, F. Ferroni ${ }^{7)}$, E. Fiorini ${ }^{1)}$, C. Gargiulo ${ }^{7)}$, E. Guardincerri ${ }^{11)}$, A. Giuliani ${ }^{12)}$, P. Gorla ${ }^{1), 8)}$, T. D. Gutierrez ${ }^{6}$, E. E. Haller ${ }^{6),}$ 10), I. G. (rastorza $^{8)}$, E. Longo ${ }^{7)}$, G. Maier ${ }^{2)}$, R. Maruyama ${ }^{6)}{ }^{10)}$, S. Morganti ${ }^{7)}$, S. Nisi ${ }^{4}$, C. Nones ${ }^{1)}$, E. B. Norman ${ }^{13)}$, A. Nucciotti ${ }^{1)}$, E. Olivieri ${ }^{5)}$, P. Ottonello ${ }^{11)}$, M. Pallavicini ${ }^{11)}$, E. Palmieri ${ }^{14)}$, M. Pavan ${ }^{1)}$, M. Pedretti ${ }^{12)}$, G. Pessina ${ }^{1)}$, S. Pirro ${ }^{1)}$, E. Previtali ${ }^{1)}$, B. Quiter ${ }^{6)}$, 10), L. Risegari ${ }^{5)}$, C. Rosenfeld ${ }^{3)}$, S. Sangiorgio ${ }^{12)}$, M. Sisti ${ }^{1)}$, A. R. Smith ${ }^{6)}$, L. Torres ${ }^{1)}$, G. Ventura ${ }^{5)}$, N. Xu ${ }^{13)}$, and L. Zanotti ${ }^{1}$ CUORE Collaboration

\begin{abstract}
CUORICINO is an array of $62 \mathrm{TeO}_{2}$ bolometers with a total mass of $40.7 \mathrm{~kg}\left(11.2 \mathrm{~kg}\right.$ of $\left.{ }^{130} \mathrm{Te}\right)$, operated at about $10 \mathrm{mK}$ to search for $\beta \beta(0 \nu)$ of ${ }^{130} \mathrm{Te}$. The detectors are organized as a 14 -story tower and intended as a slightly modified version of one of the 19 towers of the CUORE project, a proposed tightly packed array of $988 \mathrm{TeO}_{2}$ bolometers (741 kg of total mass of $\mathrm{TeO}_{2}$ ) for ultralow-background searches on neutrinoless double-beta decay, cold dark matter, solar axions, and rare nuclear decays. Started in April 2003 at the Laboratori Nazionali del Gran Sasso (LNGS), CUORICINO data taking was stopped in November 2003 to repair the readout wiring system of the 62 bolometers. Restarted in spring 2004, CUORICINO is presently the most sensitive running experiment on neutrinoless double-beta decay. No evidence for $\beta \beta(0 \nu)$ decay has been found so far and a new lower limit, $T_{1 / 2}^{0 \nu} \geq 1.8 \times 10^{24} \mathrm{yr}(90 \%$ C.L.), is set, corresponding to $\left\langle m_{\nu}\right\rangle \leq 0.2-1.1 \mathrm{eV}$, depending on the theoretical nuclear matrix elements used in the analysis. Detector performance, operational procedures, and background analysis results are reviewed. The expected performance and sensitivity of CUORE is also discussed.
\end{abstract}

\section{INTRODUCTION}

The existence of neutrino oscillations, well proven in recent years by the results of neutrino oscil-

* The text was submitted by the authors in English.

${ }^{1)}$ Dipartimento di Fisica dell'Università di Milano-Bicocca e Sezione di Milano dell'INFN, Milano, Italy.

${ }^{2)}$ Dipartimento di Ingegneria Strutturale del Politecnico di Milano, Milano, Italy.

${ }^{3)}$ Department of Physics and Astronomy, University of South Carolina, Columbia, USA.

${ }^{4)}$ Laboratori Nazionali del Gran Sasso, Assergi (L'Aquila), Italy.

${ }^{5)}$ Dipartimento di Fisica dell'Università di Firenze e Sezione di Firenze dell'INFN, Firenze, Italy.

${ }^{6)}$ Lawrence Berkeley National Laboratory, Berkeley, USA.

7) Dipartimento di Fisica dell'Università di Roma e Sezione di Roma 1 dell'INFN, Roma, Italy. lation experiments, has stimulated great interest in neutrinoless double-beta decays. By demonstrating the finiteness of neutrino mass and mixing, neutrino oscillations have in fact provided us with the first clear evidence of phenomena beyond the reach of

${ }^{8)}$ Laboratorio de Fisica Nuclear y Alta Energias, Universitad de Zaragoza, Zaragoza, Spain.

${ }^{9)}$ Kamerling Onnes Laboratory, Leiden University, Leiden, the Netherlands.

10) University of California, Berkeley, USA.

${ }^{11)}$ Dipartimento di Fisica dell'Università di Genova e Sezione di Genova dell'INFN, Genova, Italy.

${ }^{12)}$ Dipartimento di Fisica e Matematica dell'Università dell'Insubria e Sezione di Milano dell'INFN, Como, Italy.

${ }^{13)}$ Lawrence Livermore National Laboratory, Livermore, USA.

${ }^{14)}$ Laboratori Nazionali di Legnaro, Padova, Italy.

** E-mail: Oliviero.Cremonesi@mib.infn.it 
the Standard Model (SM). Other unsolved questions concerning neutrino properties have since become a subject of increasing interest and are considered a unique tool to see what new physics lies beyond $\mathrm{SM}$ predictions. We know in fact that neutrinos are massive, but we still ignore the absolute scale of their masses. Neutrino oscillations in fact depend only on the absolute value of the difference of the neutrino mass eigenvalues squared. Two possible hierarchies are then implied by current available data: the normal $\left(m_{1} \approx m_{2} \ll m_{3}\right)$ and the inverted hierarchy $\left(m_{1} \ll\right.$ $\left.m_{2} \approx m_{3}\right)$.

The same ignorance holds for the neutrino nature. In the SM, neutrinos are Dirac particles by construction (i.e., in order to conserve lepton number $L$ ). In the limit of vanishing masses, however, lepton number conservation can be equivalently stated in terms of neutrino helicity properties and the Majorana or Dirac descriptions for the neutrino are equivalent (i.e., they do not change the physical content of the theory). For finite neutrino masses, however, the two descriptions are no longer equivalent and can give rise to different physical scenarios (e.g., mass generation mechanisms).

Only experiments sensitive to $m_{1}$ can aim at solving the mass hierarchy problem. This is the case for kinematic measurements of the $\beta$-spectrum end point, $\beta \beta(0 \nu)$, and cosmological measurements. The sum of the masses of the neutrinos of the three flavors is constrained to values from 0.7 to $1.7 \mathrm{eV}$ from the Wilkinson microwave anisotropy probe full sky microwave map together with the survey of the $2 \mathrm{dF}$ galaxy redshift [1-5]. A claim for a nonzero value of $0.64 \mathrm{eV}$ has also been proposed [6]. Although these values are more constraining than upper limits of $2.2 \mathrm{eV}$ for $m_{\nu}$ obtained so far in experiments on single-beta decay, they are strongly model dependent and therefore less robust with respect to laboratory measurements. On the other hand, the best sensitivity expected for next-generation single-beta decay experiments is of the order of $\sim 0.2 \mathrm{eV}$ (KATRIN [7] and $\mathrm{Re}-\mu$ bolometers [8]).

The measurement of neutrino masses, mixing angles, and phases and the assessment of the Dirac/Majorana character of neutrinos are going to be considered primary goals of the next-generation experiments. In this scenario, neutrinoless doublebeta decay searches play a unique role giving the possibility to probe the Majorana character of neutrinos while obtaining information on the neutrino mass hierarchy and Majorana phases. If neutrinos are Majorana particles, more stringent constraints, or a positive value for the effective neutrino mass, can be obtained.
Double-beta decay (DBD) can occur in a number of even-even nuclei and can be experimentally studied for a consistent fraction of them for which singlebeta decay to the intermediate nucleus is energetically forbidden. Its transition rate can be written as

$$
\lambda_{\beta \beta}^{0 \nu}=G^{0 \nu}\left(E_{0}, Z\right)\left|\left\langle m_{\nu}\right\rangle\right|^{2}\left|M_{\mathrm{F}}^{0 \nu}-\left(g_{A} / g_{V}\right)^{2} M_{\mathrm{GT}}^{0 \nu}\right|^{2},
$$

where $G^{0 \nu}$ is the phase-space factor, $M_{\mathrm{F}}^{0 \nu}$ and $M_{\mathrm{GT}}^{0 \nu}$ are the Fermi and Gamow-Teller nuclear matrix elements, and $g_{A}$ and $g_{V}$ are the axial-vector and vector weak coupling constants. $\left\langle m_{\nu}\right\rangle$ is the effective Majorana electron neutrino mass, which can be expressed in terms of the elements of the neutrino mixing matrix as follows:

$$
\left\langle m_{\nu}\right\rangle \equiv\left|U_{e 1}^{L}\right|^{2} m_{1}+\left|U_{e 2}^{L}\right|^{2} m_{2} e^{i \phi_{2}}+\left|U_{e 3}^{L}\right|^{2} m_{3} e^{i \phi_{3}},
$$

where $e^{i \phi_{2}}$ and $e^{i \phi_{3}}$ are the Majorana $C P$ phases ( \pm 1 for $C P$ conservation), $m_{1,2,3}$ are the Majorana neutrino mass eigenvalues, and the coefficients $U_{e j}^{L}$ are the elements of the first row of the PontecorvoMaki-Nakagawa-Sakata (PMNS) neutrino mixing matrix. Taking into account the results of all oscillation experiments [9-18], we have then

$$
\begin{gathered}
\left|\left\langle m_{\nu}\right\rangle\right|=\mid(0.70 \pm 0.030) m_{1} \\
+(0.30 \pm 0.030) m_{2} e^{i \phi_{2}}+(<0.05) m_{3} e^{i \phi_{3}} \mid,
\end{gathered}
$$

which, in the case of the inverted hierarchy, implies that $\left|\left\langle m_{\nu}\right\rangle\right|$ could have a minimum value as large as $0.055 \mathrm{eV}$. This is a very important result for $\beta \beta(0 \nu)$ since it implies that next-generation experiments could have the required sensitivity to probe the inverted mass hierarchy.

Equation ( 1 ) for the $\beta \beta(0 \nu)$ rate implies an explicit dependence on the square of the nuclear matrix elements whose calculation is still quite uncertain. This is one of the most severe problems in $\beta \beta(0 \nu)$ result interpretation. As a consequence, it is imperative to search for $\beta \beta(0 \nu)$ in different nuclei. Another reason for this request is an intrinsic weakness of the $\beta \beta(0 \nu)$ signature in the calorimetric approach: in fact, the sharp line at the transition energy could in principle be mimicked by a radioactive background line. Only the discovery of the transition lines at the energies expected for $\beta \beta(0 \nu)$ in two or more candidate nuclei would therefore definitely prove its existence.

No evidence has been obtained for $\beta \beta(0 \nu)$ so far, with the only exception of an indication reported by a subset of the Heidelberg-Moscow Collaboration headed by Klapdor-Kleingrothaus [19, 20] which, in our opinion, needs, however, a deeper and more statistically significant verification, possibly through the study of candidate isotopes different from ${ }^{76} \mathrm{Ge}$. 

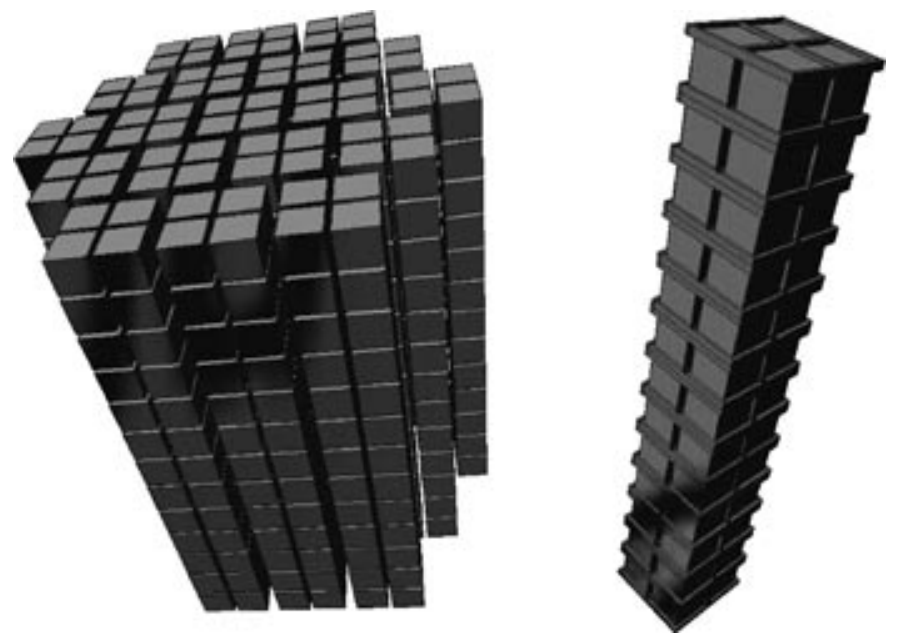

Fig. 1. Scheme of the CUORE array (left) and of one of the 19 towers (right).

Lacking any evidence for $\beta \beta(0 \nu)$ in all the experimentally studied candidate isotopes, only upper limits on its half-life can be obtained. These limits directly translate, through the nuclear matrix elements evaluations, into lower limits of the order of $0.3-1 \mathrm{eV}$ on $\left\langle m_{\nu}\right\rangle$ [21]. On the other hand, the above-cited theoretical considerations on the neutrino properties based on present results coming from atmospheric, reactor, and solar neutrino experiments seem to indicate that $\left\langle m_{\nu}\right\rangle$ should lie in the range between 0.1 and $1 \mathrm{eV}$ [22]. This reinforces the importance of designing and building experiments that could explore this mass range, and indeed several second-generation experiments (e.g., EXO, GENIUS, MAJORANA, MOON, and CUORE) [21] have been proposed during the last few years to search for the $\beta \beta(0 \nu)$ decay of various nuclei. The challenge of these experiments consists in the construction of a large mass (of the order of tons) counting facility with extremely low background. This can be easily deduced from the following simplified expression for the $1 \sigma$ sensitivity:

$$
S_{1 \sigma}^{0 \nu}=\ln (2) N_{A} \epsilon \eta \sqrt{M T} \begin{aligned}
& B \Delta \\
& B \Delta
\end{aligned}
$$

from which the explicit dependence on the relevant experimental parameters (e.g., detector mass $M$, measurement time $T$, background rate $B$, energy resolution $\Delta$, detection efficiency $\epsilon$, and isotropic abundance $\eta$ ) is apparent.

Another DBD mode, characterized by the emission of two neutrinos $(\beta \beta(2 \nu))$, is usually considered in $\beta \beta$ searches. Although allowed in the framework of the Standard Electroweak Model (second-order transition) and characterized by very long half-lives (of the order of $10^{19}-10^{20} \mathrm{yr}$ ), $\beta \beta(2 \nu)$ still represents one of the primary goals of $\beta \beta$ experiments (e.g.,
NEMO3) since it could represent a unique tool to test nuclear matrix calculations [23].

\section{CUORE}

The CUORE Project [24-26] aims at the construction of a DBD experiment based on the lowtemperature detector (LTD) technique and characterized by an active mass of the order of $1 \mathrm{t}$. The main advantages of the LTD approach with respect to other conventional techniques (e.g. Ge diodes) are a sensitivity to low- or nonionizing events and a material choice flexibility. Besides allowing an experimental investigation on any DBD active isotope, LTDs allow therefore a $\beta \beta(0 \nu)$ experimental study based on the use of materials characterized by a large natural isotopic abundance (a crucial parameter showing a linear dependence in the DBD lifetime sensitivity).

The CUORE setup (Fig. 1) will consist of 988 natural $\mathrm{TeO}_{2}$ bolometers arranged in a cylindrical configuration of 19 towers (each made of a stack of 13 fourdetector modules). Each bolometer will consist of a temperature sensor (NTD thermistor) glued to a cubic $5 \times 5 \times 5$-cm TeO ${ }_{2}$ crystal with a mass of about 750 g. ${ }^{130} \mathrm{Te}$ is in fact one of the best candidates for DBD searches. Owing to its high transition energy $(2528.8 \pm 1.3 \mathrm{keV})$, the favorable nuclear matrix elements, and the large natural isotopic abundance $(33.8 \%)$, it is possible to perform a sensitive experiment even using natural tellurium. ${ }^{130} \mathrm{Te}$ is moreover characterized by a favorable neutrinoless DBD rate and large $\mathrm{TeO}_{2}$ single crystals can be grown with excellent features as thermal detectors.

The main goal of CUORE is to reach, in the energy region of interest, a background level lower than $10^{-2}$ counts $/ \mathrm{keV} / \mathrm{kg} / \mathrm{yr}$, obtaining hence a sensitivity on the effective Majorana mass of the neutrino 
Table 1. Expected CUORE 5 -yr $\beta \beta(0 \nu)$ sensitivities under different possible performance and background scenarios $\left(\left|\left\langle m_{\nu}\right\rangle\right|\right.$ ranges are evaluated according to QRPA calculations of nuclear matrix elements)

$\begin{array}{crcc}B, \text { counts } / \mathrm{keV} / \mathrm{kg} / \mathrm{yr} & \Delta, \mathrm{keV} & T_{1 / 2}, 10^{26} \mathrm{yr} & \left|\left\langle m_{\nu}\right\rangle\right|, \mathrm{meV} \\ 0.01 & 10 & 1.5 & 23-118 \\ 0.01 & 5 & 2.1 & 19-100 \\ 0.001 & 10 & 4.6 & 13-67 \\ 0.001 & 5 & 6.5 & 11-57\end{array}$

lower than $50 \mathrm{meV}$ (Table 1). The CUORE array (Fig. 1) is designed in order to have the most compact structure, reducing to a minimum the distance between the crystals and the amount of inert material between them.

The entire array, surrounded by an ancient Roman

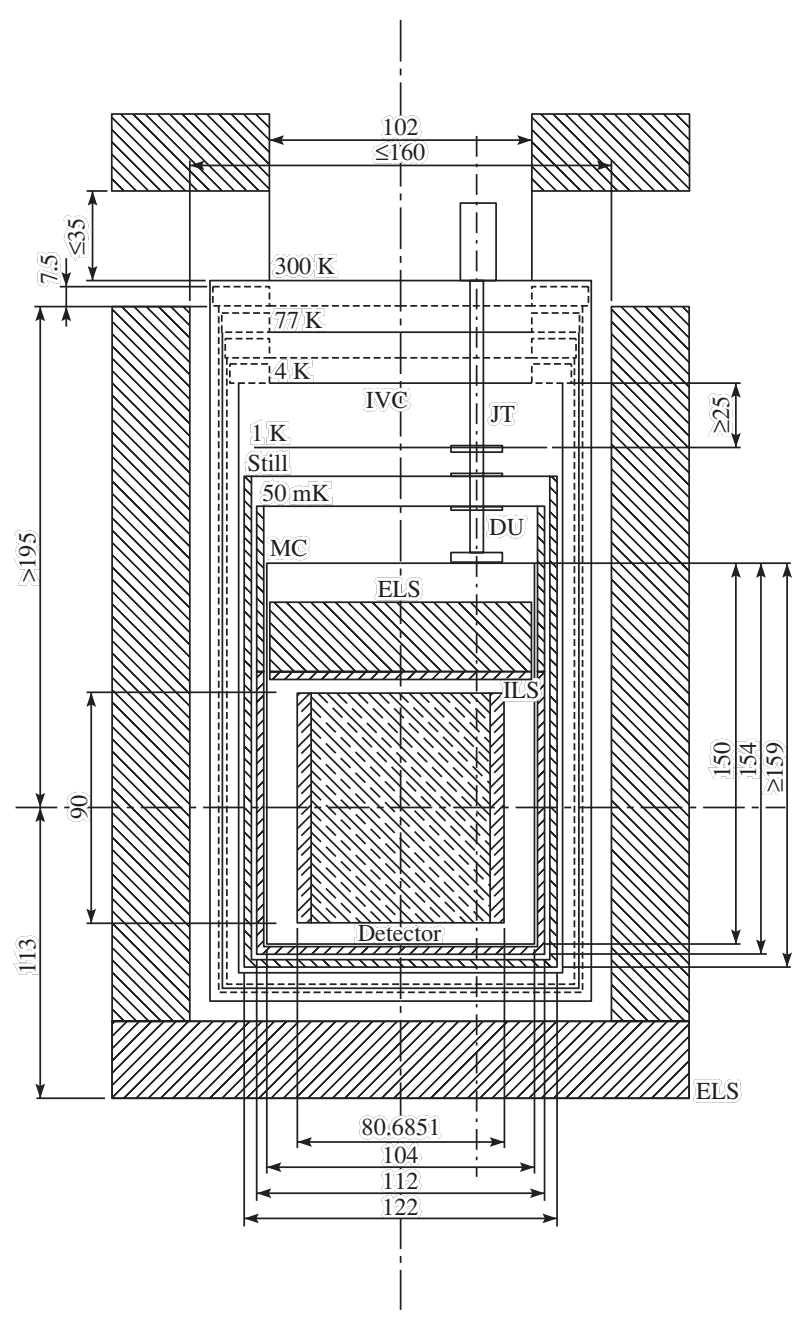

Fig. 2. Scheme of the CUORE cryostat and radiation shields (dimensions are in $\mathrm{mm}$ ). lead shield 3 to $6 \mathrm{~cm}$ thick, will be operated at about $10 \mathrm{mK}$ in a powerful ${ }^{3} \mathrm{He} /{ }^{4} \mathrm{He}$ dilution refrigerator (Fig. 2). A further thickness of $30 \mathrm{~cm}$ of low-activity lead will be used to shield the array from the dilution refrigerator setup and from the environment. A borated polyethylene shield and an air-tight cage will surround the cryostatn externally. The experiment will be installed underground in the LNGS at a depth of $3400 \mathrm{~m}$ w.e. The CUORE will be by far the largest array of low-temperature detectors ever realized.

In order to demonstrate the feasibility of such a project, an intermediate detector (named CUORICINO and consisting of a single tower of CUORE) was proposed some years ago, to be housed in the same refrigerator already used for the MiDBD experiment. Such an array, built and installed at LNGS in 2001, is now taking data (Section 3). Following the successful operation of CUORICINO, CUORE was approved in 2004 by the LNGS and INFN scientific committees. It is now under construction at LNGS in the underground experimental Hall A. First data taking is expected in 2010.

\section{CUORICINO}

The CUORICINO detector is a slightly modified version of a CUORE tower, enlarged in order to exploit all the available cryogenic experimental chamber of the dilution refrigerator installed in Hall A of LNGS. Eleven of the 13 storys of the CUORICINO tower are four-crystal modules identical to those foreseen for CUORE (Fig. 3). The remaining ones are made of nine-crystal modules, where 18 of the 20 crystals used for the MiDBD experiment have been housed. CUORICINO contains therefore $44 \mathrm{TeO}_{2}$ crystals of size $5 \times 5 \times 5 \mathrm{~cm}(790 \mathrm{~g}$ each) and $18 \mathrm{TeO}_{2}$ crystals of size $3 \times 3 \times 6 \mathrm{~cm}(340 \mathrm{~g}$ each). The total mass of $\mathrm{TeO}_{2}$ in CUORICINO is about $41 \mathrm{~kg}\left(11 \mathrm{~kg}\right.$ of $\left.{ }^{130} \mathrm{Te}\right)$. All the crystals are made of natural tellurium except for four of the smallsize ones. Two of these are isotopically enriched to $75 \%$ in ${ }^{130} \mathrm{Te}$, while the remaining two are isotopically enriched to $82.3 \%$ in ${ }^{128}$ Te. Particular care was dedicated to the selection and treatment of the materials used for the construction of the CUORICINO array: the crystals were grown with radiopure materials in China (SICCAS) and shipped to Italy, where they were optically polished with specially selected lowcontamination powders and stored underground at LNGS. The mechanical structure of the array was made exclusively of OFHC copper and PTFE, both with an extremely low radioactive content. All the copper and Teflon components of the mounting structure were separately processed with acids to remove any possible surface contamination. Finally, the array was assembled in an underground clean room, inside 

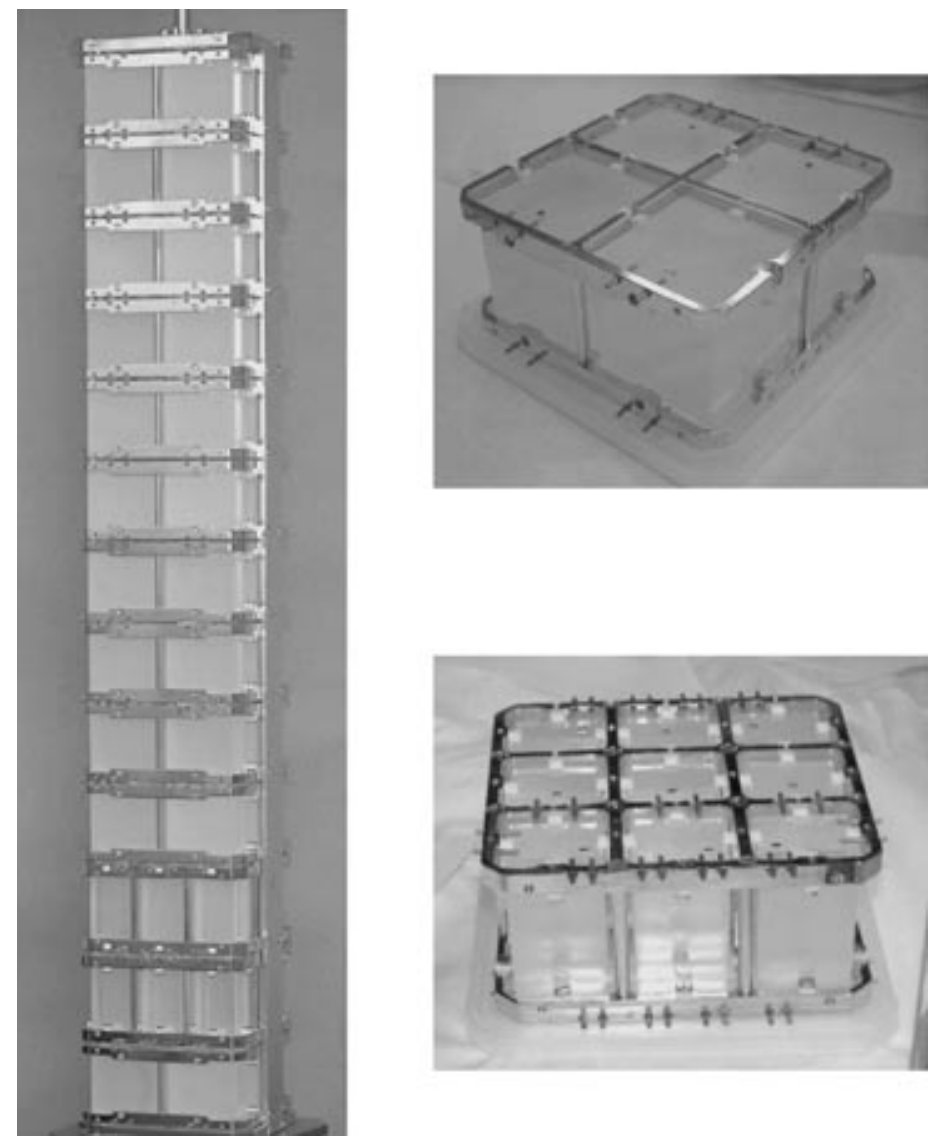

Fig. 3. The CUORICINO tower (left) and details of the two CUORICINO modules (right).

boxes flushed with clean $\mathrm{N}_{2}$ in order to avoid Rn contamination. Once closed inside its copper box, the array was mounted in the dilution refrigerator installed in Hall A of LNGS. An inner Roman lead shield kept at low temperature $(\mathrm{a} \sim 1-\mathrm{cm}$-thick tube with bottom (top) discs 7.5 (10) cm thick) completely surrounds the detector. The refrigerator itself is shielded with a $20-\mathrm{cm}$ thickness of low-activity lead and a $10-\mathrm{cm}$ thickness of borated PET. Nitrogen from a dedicated evaporator is flushed between the external lead shield and the cryostat to avoid any Rn contribution to the detector background.

CUORICINO was cooled at the beginning of year 2003. After a stop in November 2003 to fix a technical problem responsible for the disconnection of part of the readout wires, data taking restarted in spring 2004 with the full operation of all the detectors. Total statistics of 1.04 and $3.84 \mathrm{~kg}$ yr of ${ }^{130} \mathrm{Te}$ were collected in the 2003 (Run 1) and 2004 runs (Run 2, up to April 3, 2004). Starting from October 2004, the original embedded He liquefier was disconnected and manual He refilling started. Since then, the average duty cycle has improved abruptly to about $64 \%$. Data taking is currently divided in background runs lasting about four weeks, each starting and ending with a 23 d calibration with a gamma source (a ${ }^{232}$ Th source placed in contact with the outer vacuum chamber of the cryostat). A number of gamma lines due to

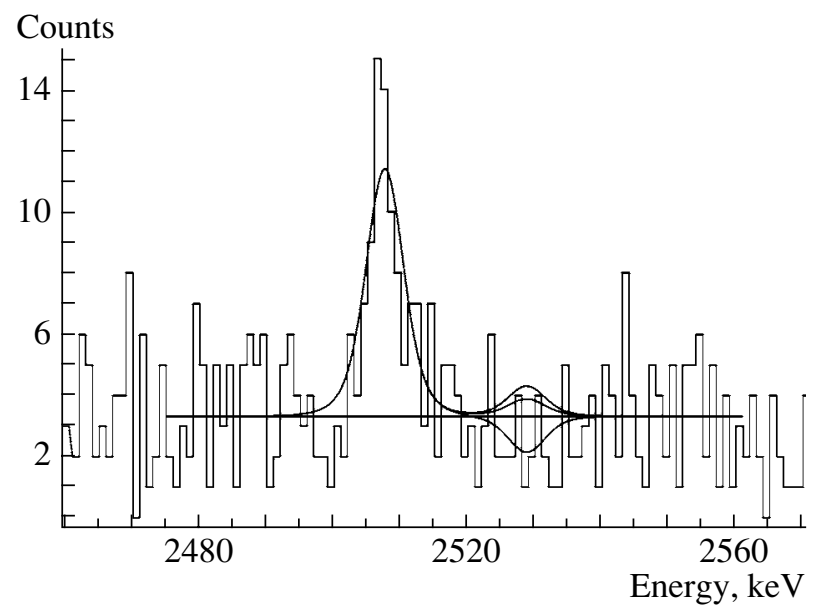

Fig. 4. Summed background spectrum from all the operating CUORICINO crystals in the region of $\beta \beta(0 \nu)$ of ${ }^{130} \mathrm{Te}(\beta \beta(0 \nu)$ best fit, 68 and $90 \%$ C.L. curves are shown). 
Table 2. Effective Majorana mass of the electron neutrino $\left|\left\langle m_{\nu}\right\rangle\right|$ corresponding to $T_{1 / 2}^{0 \nu}\left({ }^{130} \mathrm{Te}\right)=1.8 \times 10^{24} \mathrm{yr}$ derived from various nuclear ( $\mathrm{QRPA})$ models

\begin{tabular}{llc} 
Reference, author, year & \multicolumn{1}{c}{ Method } & $\left\langle m_{\nu}\right\rangle, \mathrm{eV}$ \\
[28], Staudt et al., 1992 & Pairing (Paris) & $0.21-0.22$ \\
& Pairing (Bonn) & $0.22-0.24$ \\
[29], Pantis et al., 1996 & No $p-n$ pairing & 0.66 \\
& $p-n$ pairing & 1.05 \\
[30], Vogel et al., 1986 & & 0.61 \\
[31], Civitarese et al., 1987 & & 0.54 \\
[32], Tomoda, 1991 & & 0.54 \\
[33], Barbero et al., 1999 & & 0.43 \\
[34], Simkovic, 1999 & pn RQRPA & 0.88 \\
[35], Suhonen et al., 1992 & & 0.83 \\
[36], Muto et al., 1989 & & 0.51 \\
[37], Stoica et al., 2001 & Large basis & 0.77 \\
& Short basis & 0.72 \\
[38], Faessler et al., 1998 & & 0.72 \\
[39], Engel et al., 1989 & Seniority & 0.37 \\
[40], Aunola et al., 1998 & WS & 0.50 \\
& AWS & 0.54
\end{tabular}

${ }^{60} \mathrm{Co}$ and ${ }^{40} \mathrm{~K}$ and of the ${ }^{238} \mathrm{U}$ and ${ }^{232} \mathrm{Th}$ chains are clearly visible in the background spectrum obtained in operating all the detectors in anticoincidence. It should be stressed that these lines are not visible in the spectra of the single detectors: they grow only by summing the spectra from all the detectors and are a good check of the calibration and stability of the detectors along the background measurement. The ${ }^{208} \mathrm{Tl}$ gamma line at $2615 \mathrm{keV}$, being clearly visible in the background summed spectrum, is used to evaluate the energy resolution in the $\beta \beta(0 \nu)$ region. The FWHM measured value is $7 \mathrm{keV}$ for the $5 \times$ $5 \times 5$-cm bolometers and $12 \mathrm{keV}$ for the $3 \times 3 \times 6$ $\mathrm{cm}$ ones. Smaller values are measured at lower energies (3.6- and 4.4-keV FWHM at the 120- and 583 -keV lines for the $5 \times 5 \times 5$-cm bolometers). The background rate in the region of interest for $\beta \beta(0 \nu)$ is $0.18 \pm 0.02$ counts $/ \mathrm{keV} / \mathrm{kg} / \mathrm{yr}$. No peak appears (Fig. 4) at the ${ }^{130}$ Te double-beta decay transition energy $(2528.8 \mathrm{keV})$. A maximum likelihood procedure used to establish the maximum number of $\beta \beta(0 \nu)$ events compatible with the measured background allows one to set an upper limit on the ${ }^{130} \mathrm{Te} \beta \beta(0 \nu)$ half-life of $1.8 \times 10^{24} \mathrm{yr}$ at $90 \%$ C.L.
The corresponding upper bound on the Majorana effective mass ranges from 0.2 and $1.1 \mathrm{eV}$ depending on the nuclear model used to interpret the data [27] (see Table 2).

\section{CUORICINO RESULTS AND CUORE PERSPECTIVES}

While being a self-consistent experiment on $\beta \beta(0 \nu)$ (actually the most sensitive currently running), CUORICINO must be considered as a first step towards CUORE. It is in fact an effective test facility intended to verify the technical feasibility of the parent project. Indeed the good results concerning the detector performance obtained with CUORICINO show that the construction of CUORE could be more straightforward than expected. The results of the ongoing $\mathrm{R} \& \mathrm{D}$ actually show that the increase in the number of bolometers and in the total mass do not substantially affect the experimental sensitivity. They also show that the mass of the inert materials (mainly copper and PTFE) can be reduced without any loss of performance. Results of crucial importance are also being obtained for what concerns background sources [27]. CUORICINO data analysis shows in fact that a dominant contribution to the background level originates from radioactive contaminations of the $\mathrm{TeO}_{2}$ crystals and $\mathrm{Cu}$ holders distributed over a shallow layer whose thickness is of the order of a few micrometers. This clearly results from the comparison with detailed Monte Carlo simulations, but is also apparent from the naive observation that the background rate does not decrease above the $2615-\mathrm{keV}$ line of ${ }^{208} \mathrm{Tl}$. An intensive program aimed at the reduction of such a contribution, based on dedicated surface cleaning procedures, has therefore been started. Active methods aimed at identifying events originating from surface events have also been successfully developed [41].

On the other hand, the residual contribution observed in CUORICINO and attributed to "external" gamma sources (cryostat setup and radiation shields) should be a minor problem for CUORE. Indeed, the CUORE lead shield design was optimized in order to practically cancel the background coming from outside contributions (this optimization was not possible in CUORICINO, which had to be housed in a preexisting cryostat).

The surface processing of the CUORICINO $\mathrm{TeO}_{2}$ crystals and mounting structure (copper and PTFE) was recently improved in the framework of the abovementioned $\mathrm{R} \& \mathrm{D}$. A reduction by approximately a factor of 5 in the $\beta \beta(0 \nu)$ background contribution from the crystal surface was in fact obtained by a careful cleaning of the $\mathrm{TeO}_{2}$ crystal surface by means of radioclean powders and chemicals. More 
detailed knowledge on the background origin and location is expected from the systematic study of the CUORICINO background with larger statistics (single crystal background distributions, time coincidences, etc.). It will definitely help us in disentangling the background sources and finally reducing the CUORE background level below the sought level of 0.01 counts $/ \mathrm{keV} / \mathrm{kg} / \mathrm{yr}$.

\section{CONCLUSIONS}

With a 5 -yr sensitivity of about $10^{25} \mathrm{yr}(68 \%$ C.L.) for the ${ }^{130} \mathrm{Te} \beta \beta(0 \nu)$, CUORICINO is the presently running experiment with the best sensitivity on the effective Majorana neutrino mass $\left\langle m_{\nu}\right\rangle$ (100-500 meV, according to extreme nuclear matrix evaluations in [28] and [29], respectively). Moreover, thanks to the CUORICINO results, the LTD technology is now proven and a second-generation experiment (CUORE) is under construction at LNGS. It will represent by far the most cost-effective nextgeneration DBD experiment able to reach the desired levels of sensitivity to probe the neutrino mass inverted hierarchy.

\section{ACKNOWLEDGMENTS}

This work has been partially supported by the Commission of European Communities (contract no. HPRN-CT-2002-00322), by the US Department of Energy (contract no. DEAC03-76-SF00098), and by the National Science Foundation (grant no. PHY-0139294).

\section{REFERENCES}

1. V. Barger et al., Phys. Lett. B 595, 55 (2004).

2. S. Hannestad et al., J. Cosmol. Astropart. Phys. 05, 004 (2003).

3. M. Tegmark et al., Phys. Rev. D 69, 103501 (2004).

4. D. N. Spergel et al., Astrophys. J. Suppl. 148, 175 (2003).

5. P. Crotty et al., Phys. Rev. D 69, 123007 (2004).

6. S. W. Allen et al., Mon. Not. R. Astron. Soc. 346, 593 (2003).

7. V. M. Lobashev, Nucl. Phys. A 719, 153 (2003), and references therein.

8. C. Arnaboldi et al., MARE, Milano Int. Note (Spring 2005).

9. F. Feruglio et al., Nucl. Phys. B 637, 345 (2002).

10. F. Feruglio et al., Nucl. Phys. B 659, 359 (2003).

11. F. Joaquim, Phys. Rev. D 68, 033019 (2003).

12. C. Giunti, hep-ph/0308206.

13. S. Pascoli and S. T. Petkov, Phys. Lett. B 544, 239 (2002).
14. S. Pascoli and S. T. Petkov, Phys. Lett. B 580, 280 (2004).

15. J. Bahcall and C. Pena-Garay, J. High Eenergy Phys. 0311, 004 (2003).

16. J. Bahcall et al., Phys. Rev. D 70, 033012 (2004).

17. H. Murayama and C. Pena-Garay, Phys. Rev. D 69, 031301 (2004).

18. J. Suhonen and O. Civitarese, Phys. Rep. 300, 123 (1998).

19. H. V. Klapdor-Kleingrothaus, A. Dietz, I. V. Krivosheina, and O. Chkvorets, Nucl. Instrum. Methods Phys. Res. A 522, 371 (2004).

20. H. V. Klapdor-Kleingrothaus, A. Dietz, I. V. Krivosheina, et al., Phys. Lett. B 578, 54 (2004).

21. S. R. Elliot and P. Vogel, Annu. Rev. Nucl. Part. Sci. 52, 115 (2002); hep-ph/0202264.

22. F. Feruglio, A. Strumia, and F. Vissani, Nucl. Phys. B 637, 345 (2002).

23. A. Faessler et al., in Proceedings of the MEDEX'03 Conference, Prague, Czech Republic, 2003.

24. E. Fiorini and T. Niinikoski, Nucl. Instrum. Methods 224, 83 (1984).

25. C. Arnaboldi et al., Nucl. Instrum. Methods Phys. Res. A 518, 774 (2004); hep-ex/0212053.

26. C. Arnaboldi et al., Astropart. Phys. 20, 91 (2003); hep-ex/0302021.

27. CUORE Collab., CUORE Proposal to the LNGS and INFN Scientific Committees, hep-ex/0501010; http://crio.mib.infn.it/wig/Cuorepage.

28. A. Staudt, T. T. S. Kuo, and H. V. KlapdorKleingrothaus, Phys. Rev. C 46, 871 (1992).

29. G. Pantis, F. Simkovic, J. D. Vergados, and A. Faessler, Phys. Rev. C 53, 695 (1996).

30. P. Vogel et al., Phys. Rev. Lett. 57, 3148 (1986); P. Vogel et al., Phys. Rev. C 37, 73 (1988); M. Moe and P. Vogel, Annu. Rev. Nucl. Part. Sci. 44, 247 (1994).

31. O. Civitarese, A. Faessler, and T. Tomoda, Phys. Lett. B 194, 11 (1987); T. Tomoda and A. Faessler, Phys. Lett. B 199, 473 (1987); J. Suhonen and O. Civitarese, Phys. Rev. C 49, 3055 (1994).

32. T. Tomoda, Rep. Prog. Phys. 54, 53 (1991).

33. C. Barbero et al., Nucl. Phys. A 650, 485 (1999).

34. F. Simkovic, Phys. Rev. C 60, 055502 (1999).

35. J. Suhonen, O. Civitarese, and A. Faessler, Nucl. Phys. A 543, 645 (1992).

36. K. Muto, E. Bender, and H. V. Klapdor, Z. Phys. A 334, 187 (1989)

37. S. Stoica and H. V. Klapdor, Phys. Rev. C 63, 064304 (2001).

38. A. Faessler and F. Simkovic, J. Phys. G 24, 2139 (1998).

39. J. Engel et al., Phys. Lett. B 225, 5 (1989).

40. M. Aunola and J. Suhonen, Nucl. Phys. A 643, 207 (1998).

41. L. Foggetta et al., Appl. Phys. Lett. 86, 134106 (2005). 